\title{
EFEITOS DE 16 SEMANAS DE TREINAMENTO AERÓBIO INTERVALADO PERIODIZADO SOBRE VALORES DE GLICEMIA EM INDIVÍDUOS COM SÍNDROME METABÓLICA
}

\author{
Alan Marcel Milanez ${ }^{1}$, Stephanie Nogueira Linares ${ }^{2}$, Rodolfo Augusto Travagin Miranda ${ }^{2}$, Carlos Ivan Mesa ${ }^{2}$, \\ Maria Paula Ferreira de Figueiredo², Carlos Marcelo Pastre ${ }^{2}$, Jayme Netto Júnior². \\ Universidade Estadual Paulista - UNESP, ${ }^{1}$ Departamento de Educação Física; ${ }^{2}$ Departamento de Fisioterapia, \\ Presidente Prudente, SP. e-mail: ste linares@hotmail.com \\ Agência de fomento: FAPESP.
}

\section{RESUMO}

A Síndrome Metabólica (SM) é conhecida como um conjunto de fatores de risco (hipertensão, hiperglicemia, dislipidemia e obesidade abdominal), os quais estão associados ao desenvolvimento de diabetes melitus tipo II, doença cardiovascular e morte prematura. O objetivo foi analisar os efeitos de um Treinamento aeróbio intervalado (TAI) periodizado sobre níveis glicêmicos em indivíduos com SM. Participaram do estudo 20 pessoas, com idade entre 35 e 60 anos de ambos os sexos. Realizaram-se análises bioquímicas e antropométricas nos momentos pré e pós intervenção. Não houve diferença estatística significativa na comparação entre e intra grupos para a variável glicemia, peso e índice de massa corpórea (IMC). Embora recomenda-se a prática regular de exercício físico para o controle glicêmico, o TAl, sem controle alimentar, apresentou apenas manutenção da glicemia de jejum. Conclui-se que 13 semanas de TAI periodizado não foi capaz de modificar os níveis de glicemia de jejum em indivíduos com SM.

Palavra-chave: Síndrome $X$ metabólica, exercício, estilo de vida sedentário, tratamento aeróbio, diabetes Mellitus.

\section{EFFECTS OF 16 WEEKS OF A AEROBIC INTERVAL TRAINING AEROBIC PERIODIZED ON BLOOD GLUCOSE VALUES AMONG PEOPLE WITH METABOLIC SYNDROME}

\begin{abstract}
Metabolic syndrome is a cluster of risk factors (hypertension, dyslipidemia, abdominal obesity and hyperglycemia) for cardiovascular disease, type 2 diabetes mellitus and premature death. The objective was to analyse the effects of aerobic interval training (AIT) on blood glucose levels in pacients with Metabolic syndrome. Biochemical and anthropometric analysis was measured in twenty participants (age between 35-60 years) of both genders at pre e post intervention moments. There were no statistically significant difference within and between groups for blood glucose levels, weigth and body mass index (BMI). Although it's recommended to improve physical exercise for glycemic control, AIT without diet intervention showed only maintenance of fasting glucose. Thirteen weeks of AIT was not able to modify metabolic and anthropometric variables in pacients with metabolic syndrome.
\end{abstract}

Keywords: Metabolic syndrome X, exercise, sedentary lifestyle, aerobic treatment, diabetes mellitus.

\section{INTRODUÇÃO}

A Síndrome Metabólica (SM) é caracterizada por um conjunto de indicadores de riscos como hipertensão, hiperglicemia, dislipidemia e obesidade abdominal ${ }^{1-4}$.

A causa subjacente da SM continua a desafiar os especialistas, no entanto, a resistência à insulina e obesidade visceral são postuladas como os principais componentes. Predisposição genética, sedentarismo, tabagismo, padrão alimentar e alterações hormonais podem também ter efeito causal sobre a $\mathrm{SM}^{1-3}$.

Indivíduos com SM são suscetíveis ao desenvolvimento de doenças cardiovasculares (DC), Diabetes Mellitus Tipo II (DM2) e consequentemente risco de morte prematura ${ }^{2}$. Sabe-se que a DM2 é uma doença metabólica com alta prevalência no mundo, e elevadas taxas 
de morbidade e mortalidade. Estima-se que em 2030 haja 552 milhões de pessoas com DM2 em todo mundo.

Estudos epidemiológicos demonstram relação entre inatividade física e nutrição desbalanceada aos fatores de risco. Diferentes associações de saúde como American College of Sports Medicine, Center for Disease Control and Prevention, American Heart Association, National Institutes of Health, US Surgeon General, Sociedade Brasileira de Cardiologia $^{11}$, entre outras, recomendam a prática regular de atividade física como forma de prevenção e reabilitação de doenças cardiovasculares e outras doenças crônicas ${ }^{4}$.

Pesquisadores relatam efeitos benéficos da prática regular de atividade física com relação à hipertensão arterial, resistência à insulina, DM2, dislipidemia e obesidade. Desta forma, o exercício físico pode controlar o progresso de doenças crônicas relacionadas à idade e ao estilo de vida, atuando no aumento do gasto calórico, ganho de massa muscular e diminuição de gordura hepática $^{3-5}$.

Mediante o exposto, o presente estudo tem como objetivo analisar os efeitos do treinamento aeróbio intervalado (TAI) periodizado sobre níveis glicêmicos em indivíduos com SM.

\section{METODOLOGIA}

\section{População do estudo}

O estudo caracteriza-se por um ensaio clínico randomizado. A população é constituída por participantes de ambos os sexos diagnosticados com SM, idade entre 35 e 60 anos e sedentários por no mínimo 6 meses, sendo o nível de atividade física mensurado pelo questionário de Baecke ${ }^{6}$. A definição de SM de acordo com a Federação Internacional de Diabetes será adotada ${ }^{7}$.

Os sujeitos foram informados sobre os procedimentos e objetivos da pesquisa, e após concordarem, assinaram o termo de consentimento livre e esclarecido, onde ficou assegurada a privacidade dos mesmos, bem como, o anexo de uma cópia do atestado médico, Ihes assegurando condição física para realização de exercícios. $O$ presente projeto está aprovado pelo comitê de ética sob o número de CAAE: 31687114.8.0000.5402.

\section{Randomização}

Os participantes foram alocados de maneira aleatória em 2 grupos, grupo treinamento aeróbio intervalado $(\mathrm{TAl}=10) \mathrm{e}$ grupo controle $(G C=10)$, o qual não foi submetido à nenhum tipo de intervenção.

O grupo treinamento realizou 39 sessões, 3 vezes por semana, durante o período de 16 semanas. Todos os participantes foram orientados a manterem dieta e atividades diárias normalmente durante este período.

\section{Treinamento aeróbio intervalado}

As dinâmicas de treinamento ocorreram no setor de cardiologia em um centro de fisioterapia e reabilitação na UNESP, em Presidente Prudente.

Esteiras ergométricas das marcas Movement ${ }^{\circledR}$ (modelos profissional LX-160 e profissional LX-170) e Inbramed ${ }^{\circledast}$ (modelo Export) foram utilizadas.

Para a prescrição da intensidade de treino, foi utilizada a frequência cardíaca de reserva, de acordo com a fórmula a seguir: $F C R=$ (FCmáx - FCrep) x \% treinamento + FCrep, onde: $\mathrm{FCR}=$ frequência cardíaca de reserva; $\mathrm{FCmax}=$ frequência cardíaca máxima; FCrep = frequência cardíaca de repouso; \% treinamento = percentual de treinamento. Para o cálculo da FCmáx foi utilizada a fórmula de Karvonen, descrita por $\mathrm{FCmax}=220$ - idade (em anos) ${ }^{8,9}$; e para mensuração da FCrep foi utilizado o cardiofrequencímetro da marca Polar ${ }^{\circledR}$, utilizando a média do 5o ao 250 minuto de repouso dos participantes.

Para os pacientes que faziam o uso de betabloqueadores utilizou-se a correção para a FC de acordo com o I Consenso Nacional de Reabilitação Cardiovascular ${ }^{10}$, pela fórmula: \%FC a corrigir $=Y+95,58 / 9,74$, onde $Y$ é a dose em $\mathrm{mg}$ de propranolol ou equivalente (para a dosagem dos medicamentos equivalentes será utilizada a tabela de Kaplan) $)^{10}$. A porcentagem resultante desta fórmula era então subtraída da FCmáx e posteriormente calculado a FCR.

$O$ treinamento foi realizado na esteira com intensidade entre $20-90 \%$ da FCR (Quadro 1), segundo consenso estabelecido por diversas associações mundiais de saúde ${ }^{11}$. 
Quadro 1. Programa de treinamento periodizado.

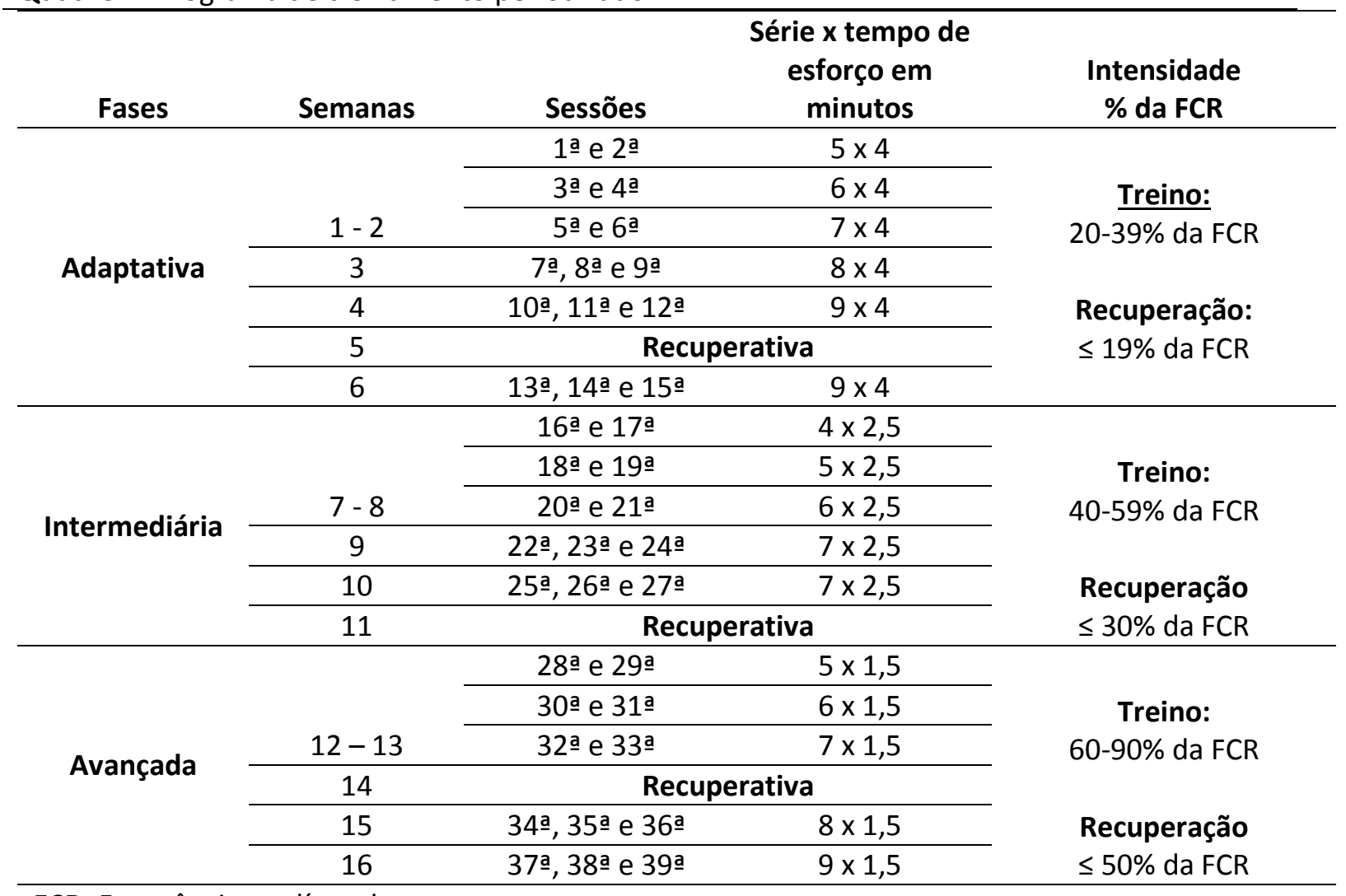

FCR: Frequência cardíaca de reserva.

\section{Análises antropométrica e glicemia de jejum}

A estatura dos foi mensuradas em posição ortostática por meio de um estadiômetro da marca Sanny ${ }^{\circledR}$ e o peso por meio de uma balança bioelétrica da marca Tanita ${ }^{\circledR}$, modelo BC -418, Segmental Body Composition Analyzer ${ }^{12}$.

Para a variável glicemia de jejum, foram realizados exames de sangue antes e após o término do treinamento, por profissional especializado. Os voluntários foram orientados a evitar qualquer atividade física vigorosa até 48 horas antecedentes à coleta e jejum de doze horas. O método de análise para glicemia de jejum foi automatizado por química seca, por meio de equipamento Fusion 5.1 FS - Ortho Clinical Diagnostic, da marca Jhonson \& Jhonson ${ }^{\circledR 13}$.

\section{Análises Estatísticas}

A normalidade dos dados foi verificada pelo teste de Shapiro-wilk. Os dados referentes às variáveis peso e IMC mostraram-se comportamento paramétrico, e a variável glicemia não paramétrico.
Test T-student, dependente e independente, foi utilizado para dados com comportamento normal. Para dados não normais, utilizou-se os testes de Wilcoxon (análise intra grupo) e Mann Whitney (análise entre grupos). Todas as conclusões foram realizadas no nível de $5 \%$ de significância.

\section{RESULTADOS}

Os dados descritivos com os valores das variáveis analisadas estão descritos na Tabela 1.

Com relação a variável bioquímica glicemia, não houve diferença estatística significativa na comparação entre e intra grupos (pré e pós intervenção), assim como para as variáveis antropométricas de peso e índice de massa corporal (IMC). Porém, observa-se que entre momentos, a variável peso corporal do GC apresenta discreto aumento em média, enquanto que, o grupo TAl demonstrou manutenção do mesmo. Nota-se o mesmo comportamento para a variável glicemia. 
Tabela 1. Valores em média e desvio padrão dos momentos pré e pós treinamento.

\begin{tabular}{lcccc}
\hline \multirow{2}{*}{ Variáveis } & \multicolumn{2}{c}{ Controle $(\mathbf{n}=\mathbf{1 0})$} & \multicolumn{2}{c}{ TAI $(\mathbf{n}=\mathbf{1 0})$} \\
\cline { 2 - 5 } & Pré & Pós & Pré & Pós \\
\hline Peso (Kg) & $84,67 \pm 18,40$ & $85,39 \pm 18,55$ & $96,01 \pm 14,10$ & $95,75 \pm 15,13$ \\
\hline IMC (Kg/m $)$ & $30,64 \pm 4,36$ & $30,89 \pm 4,38$ & $32,96 \pm 2,87$ & $32,18 \pm 3,07$ \\
\hline Glicemia $(\mathbf{m g} / \mathbf{d L})$ & $103,7 \pm 21,59$ & $114,8 \pm 39,88$ & $106,1 \pm 15,54$ & $107,8 \pm 26,76$ \\
\hline IMC = Índice de Massa Corporal; TAI = Treinamento Aeróbio Intervalado.
\end{tabular}

\section{DISCUSSÃO}

Nas últimas décadas tem havido aumento significativo da obesidade devido à alta ingestão energética e ao estilo de vida sedentário, acarretando num problema de saúde pública. Evidências sugerem que isso ocorre devido ao desequilíbrio entre baixo gasto energético e alto consumo calórico. Além do componente obesidade, estudos relatam que indivíduos com SM apresentam um risco 5 vezes maior de desenvolver $\mathrm{DM}^{2-4} \mathrm{e}$, portanto, intervenções para o controle e tratamento da SM têm sido amplamente estudado.

Em um recente estudo, Verrusio et al. ${ }^{14}$ ao analisarem os efeitos da dieta associada ao exercício físico e dieta isolada, após 6 meses de intervenção, observou que apenas os grupos que receberam treinamento físico e controle da dieta foram capazes de diminuir significativamente os valores de glicemia de jejum, divergindo dos nossos achados. Desta forma, é evidente na literatura a importância do binômio exercício físico e controle nutricional, o qual não houve no presente estudo e pode ter refletido sobre os desfechos apresentados.

No entanto, os efeitos isolados do exercício físico regular também estão relacionados a benefícios sobre os componentes da SM, tais como a circunferência abdominal, glicemia e sobre pressão arterial sistólica. Evidências comprovam que o aumento no nível de atividade física melhora os quadros de resistência insulínica e $D M 2$, além de estar inversamente associada ao desenvolvimento de fatores de risco cardiovascular. Estes efeitos podem ser explicados pela regulação da biogênese mitocondrial, adaptações sobre o tecido músculo esquelético e, principalmente, pelo efeito anti-inflamatório do exercício crônico ${ }^{15}$.
Além dos efeitos benéficos supracitados, sabe-se que o exercício físico pode também elevar a Taxa Metabólica de Repouso (TMR) devido à oxidação de substratos, aumento nos níveis de catecolaminas e estimulação de síntese proteica. No entanto, este efeito pode variar de acordo com o tipo, intensidade e duração de esforço realizado ${ }^{4,16,17}$.

Com relação às modalidades de exercício, recomenda-se a realização de exercícios aeróbios para melhora da tolerância à glicose e dos níveis de insulina em indivíduos com DM2. Embora o exercício resistido também seja uma modalidade amplamente utilizada com fins terapêuticos, uma recente revisão sistemática com meta-análise recomenda sua prática apenas para manutenção de valores de glicemia em indivíduos com $\mathrm{SM}^{3}$.

As limitações do presente estudo são representadas pelo baixo número de participantes, pelo não controle alimentar e não realização do índice HOMA, análise amplamente utilizada para avaliação da resistência insulínica ${ }^{18}$. No entanto, a ausência do controle alimentar justifica-se pelo objetivo do estudo em analisar os efeitos do exercício isolado nesta população.

\section{CONCLUSÃO}

Conclui-se que 13 semanas de treinamento aeróbio intervalado periodizado não foi capaz de modificar os valores de glicemia de jejum em indivíduos com Síndrome Metabólica.

\section{CONFLITOS DE INTERESSE}

Os autores declaram não haver qualquer potencial de conflito de interesse que possa interferir na imparcialidade deste trabalho científico.

\section{REFERÊNCIA}

1. Martins MS, Farinha JB, Benetti CB, Courtes $A A$, Duarte $T$, Silva JCN et al. Positive effects of 
resistance training on inflammatory parameters in men with metabolic syndrome risk factors. Nutricion hospitalaria. 2015;32(2):792-8.

2. Vidigal FC, Bressan J, Babio N, Salas-Salvadó J. Prevalence of metabolic syndrome in Brazilian adults: a systematic review. BMC Pub Health. 2013;13(1):1-10.

DOI:

\section{https://doi.org/10.1186/1471-2458-13-1198}

3. Lemes ÍR, Ferreira PH, Linares SN, Machado AF, Pastre CM, Netto J. Resistance training reduces systolic blood pressure in metabolic syndrome: a systematic review and meta-analysis of randomised controlled trials. $\mathrm{Br} J$ Sports Med. 2016;50:1438-42.

DOI:

https://doi.org/10.1136/bjsports-2015-094715

4. Ciolac EG, Guimarães GV. Exercício físico e síndrome metabólica. Rev Bras Med Esporte. 2004;10:319-24.

DOI:

https://doi.org/10.1590/S1517-

\section{9}

5. Kang SJ, Kim EH, Ko KJ. Effects of aerobic exercise on the resting heart rate, physical fitness, and arterial stiffness of female patients with metabolic syndrome. J Phys Ther Sci. 2016;28(6):1764-8.

DOI:

https://doi.org/10.1589/ipts.28.1764

6. Florindo AA, Latorre MdRDdO. Validation and reliability of the Baecke questionnaire for the evaluation of habitual physical activity in adult men. Rev Bras Med Esporte. 2003;9:129-35. DOI: https://doi.org/10.1590/S1517-

86922003000300002

7. Alberti KG, Eckel RH, Grundy SM, Zimmet PZ, Cleeman JI, Donato KA, et al. Harmonizing the metabolic syndrome: a joint interim statement of the International Diabetes Federation Task Force on Epidemiology and Prevention; National Heart, Lung, and Blood Institute; American Heart Association; World Heart Federation; International Atherosclerosis Society; and International Association for the Study of Obesity. Circulation. 2009;120(16):1640-5. DOI: https://doi.org/10.1161/CIRCULATIONAHA.109.1 $\underline{92644}$

8. American Association of C, Pulmonary R, American College of Cardiology F, American Heart Association Task Force on Performance $M$, Thomas RJ, King $M$, et al. AACVPR/ACCF/AHA 2010 Update: Performance Measures on Cardiac Rehabilitation for Referral to Cardiac Rehabilitation/Secondary Prevention Services Endorsed by the American College of Chest Physicians, the American College of Sports Medicine, the American Physical Therapy
Association, the Canadian Association of Cardiac Rehabilitation, the Clinical Exercise Physiology Association, the European Association for Cardiovascular Prevention and Rehabilitation, the Inter-American Heart Foundation, the National Association of Clinical Nurse Specialists, the Preventive Cardiovascular Nurses Association, and the Society of Thoracic Surgeons. J Am Coll Cardiol. 2010;56(14):1159-67.

9. Camarda SRdA, Tebexreni AS, Páfaro CN, Sasai FB, Tambeiro VL, Juliano Y, et al. Comparação da freqüência cardíaca máxima medida com as fórmulas de predição propostas por Karvonen e Tanaka. Arq Bras Cardiol. 2008;91:311-4. DOI: https://doi.org/10.1590/S0066782X2008001700005

10. Diretriz de Reabilitação Cardíaca. Arquivos Brasileiros de Cardiologia. 2005;84:431-40.

11. Mezzani A, Hamm LF, Jones AM, McBride PE, Moholdt $\mathrm{T}$, Stone JA, et al. Aerobic exercise intensity assessment and prescription in cardiac rehabilitation: a joint position statement of the European Association for Cardiovascular Prevention and Rehabilitation, the American Association of Cardiovascular and Pulmonary Rehabilitation and the Canadian Association of Cardiac Rehabilitation. Eur J Prev Cardiol. 2013;20(3):442-67.

DOI:

https://doi.org/10.1177/2047487312460484

12. Pietrobelli A, Rubiano F, St-Onge MP, Heymsfield SB. New bioimpedance analysis system: improved phenotyping with whole-body analysis. Eur J Clin Nutr. 2004;58(11):1479-84. DOI: https://doi.org/10.1038/sj.ejcn.1601993

13. Friedewald WT, Levy RI, Fredrickson DS. Estimation of the concentration of low-density lipoprotein cholesterol in plasma, without use of the preparative ultracentrifuge. Clin Chem. 1972;18(6):499-502.

14. Verrusio W, Andreozzi $P$, Renzi $A$, Martinez A, Longo $G$, Musumeci $M$, et al. Efficacy and safety of spinning exercise in middle-aged and older adults with metabolic syndrome: randomized control trial. Annali Dell'Istituto Superiore di Sanita. 2016;52(2):295-300.

15. Earnest $C P$, Johannsen NM, Swift DL, Gillison $F B$, Mikus CR, Lucia A, et al. Aerobic and strength training in concomitant metabolic syndrome and type 2 diabetes. Med Sci Sports Exerc. 2014;46(7):1293-301. DOI: https://doi.org/10.1249/MSS.0000000000000242 16. Gómez-Banoy N, Mockus I. MicroRNAs: Marcadores séricos en diabetes mellitus tipo 2 y ejercicio físico. Rev Méd Chile. 2016;144:355-63. 
DOI: $\quad$ https://doi.org/10.4067/S0034-

$\underline{98872016000300011}$

17. Alkahtani S, Elkilany A, Alhariri M. Association between sedentary and physical activity patterns and risk factors of metabolic syndrome in Saudi men: A cross-sectional study. BMC Pub Health. 2015;15:1234.

DOI:

https://doi.org/10.1186/s12889-015-2578-4

18. Keshel TE, Coker RH. Exercise Training and Insulin Resistance: A Current Review. J Obes Weight Loss Ther. 2015;5(5):S5.

Recebido para publicação em 25/08/2016

Revisado em 30/08/2016

Aceito em 30/08/2016 